\title{
Research on the interconnection and rapid deployment of smart energy service platforms under multi-factor game balance
}

\author{
Ke Qiao ${ }^{1, *}$, Xinling $\mathrm{Wu}^{2}$, Tao Zhan ${ }^{3}$, Furong Huang ${ }^{1}$, and Junchao Zhou ${ }^{1}$ \\ ${ }^{1}$ Beijing Guodiantong Network technology co., LTD, 100070, Beijing, China \\ ${ }^{2}$ State Grid Xiong'an Digital technology co, LTD, 100070, Beijing, China \\ ${ }^{3}$ State Grid Jiangxi Electric Power Co., LTD, 330077 Nanchang, China
}

\begin{abstract}
This paper firstly models the relationship between power companies, photovoltaic power generation, wind power generation networks, and users as a three-stage Steinberg game. In this game model, the power company is the dominant player and the leader of the entire game. Secondly, with the game model, a detailed analysis is carried out to obtain the optimal strategy of each game party. Finally, the simplified model was simulated and verified. The simulation result is consistent with the theoretical solution result. The research results of this paper have improved the level of energy services and innovated energy service models.
\end{abstract}

\section{Research background and its significance}

At present, a new round of energy revolution is accelerating on a global scale. Smart energy has emerged and developed rapidly, profoundly changing the production and lifestyle of human society. Smart energy, that is, an energy form that has human brain functions such as self-organization, self-inspection, self-balancing, and self-optimization, and meets the requirements of system, safety, cleanliness, and economy. It is not only the ultimate goal of the energy revolution, but also the necessary process of the energy revolution. It is regarded as the basic source of power to set off the third wave of industrial revolution. The smart energy revolution will promote the rapid development of a huge market.

Paras Sitoula has studied the context-aware smart energy recommender [1]; Quanyan Zhu. Researched the game theory model and design mechanism of deception and anti-deception[2]; Xiaotong $\mathrm{Wu}$ researched game theory about mobile location privacy[3];Pan Li researched mixed utility functions for unexpected suggestions[4]; Xiaozhong Zhang researched interactive view recommendation[5]; Changhui Yang studied the economic benefit evaluation of distributed photovoltaic energy storage hybrid system based on dynamic load;Eren Yildiz studied the defragmentation of energy storage in battery-less sensor devices; Jiasheng Zhang studied the use of independent system operators to invest and manage stored energy as public assets; Vikas Hassija studied the energy transaction framework between

\footnotetext{
* Corresponding author: qiaoke@sgitg.sgcc.com.cn
} 
solar base stations and the grid based on blockchain; But none of them use game theory to improve energy service levels and innovate energy service models.

\section{Energy research of smart energy service platform based on game theory}

This paper fully considers the relationship and interaction between power companies, thermal power generation, solar power generation, wind power generation, and users. The smart grid system composed of the three is modeled as a Steinberg game model. The users are determined by a dominant power The company and multiple photovoltaic power generation and wind power generation networks provide power to users at the same time, and users have a higher preference for the electricity sold by photovoltaic power generation and wind power generation networks based on renewable energy power generation. The total number of power companies is recorded as I, the total number of photovoltaic and wind power generation networks is recorded as $\mathrm{M}$, and the total number of users is recorded as $\mathrm{C}$. Among them, the leader of the power company game, photovoltaic power generation, wind power generation network and users are the followers of the game.[6]

This paper adopts a method based on multi-objective optimization. The utility functions of power companies, photovoltaic power generation and wind power generation networks are all modeled as electricity sales minus power generation costs and pollution emission costs, and the user's utility function modeling is user satisfaction minus purchases. Electricity expenses.

The utility function of the power company is:[7]

$$
\begin{aligned}
& U_{\mathrm{g}}\left(L_{g}, p_{g}\right) \\
& =R_{g}\left(L_{g}, p_{g}\right)-C_{g}\left(L_{g}\right)-I_{g}\left(L_{g}\right) \\
& =L_{g} p_{g}-a_{g}\left(L_{g}\right)^{2}-b_{g} L_{g}-c_{g}-\alpha_{g}\left(L_{g}\right)^{2}-\beta_{g} L_{g}
\end{aligned}
$$

In the above announcement, the meaning of each function is:

$R_{g}\left(L_{g}, p_{g}\right)$--Electricity company sales income;

$C_{g}\left(L_{g}\right)$--Electricity company generation cost;

$I_{g}\left(L_{g}\right)$--The cost of polluting emissions from power companies;

$p_{g}$--Electricity price;

$L_{g}=\sum_{c=1}^{c} L_{c, g}$ The amount of electricity purchased by all users from the power company.

The three-stage Steinberg game process is as follows:[8]

The first stage: the dominant power company, as the leader of the game, first release the electricity price $p_{g}[9]$.

The second stage: the $\mathrm{m}$-th photovoltaic power generation and wind power generation network, as a follower of the game, decides its own electricity price as $p_{m}$ according to the electricity price $p_{g}$ issued by the power company: 
The third stage: the c user, as a follower of the game, determines the power purchase $L_{c, g}$ from the power company based on the electricity price $p_{g}$ issued by the power company in the first stage and the electricity price $p_{m}$ issued by the photovoltaic power generation and wind power generation network in the second stage, The electricity purchased from mth photovoltaic power generation and wind power generation network $L_{c, m}$.

\section{Simulation analysis}

According to the mathematical model using matlab to simulate, the simulation results are as follows:

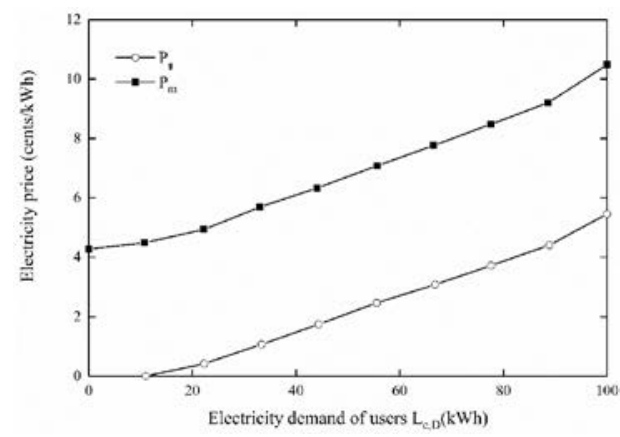

Fig. 1. The trend of electricity sales prices of power companies and photovoltaic power generation and wind power generation networks with changes in user power demand.

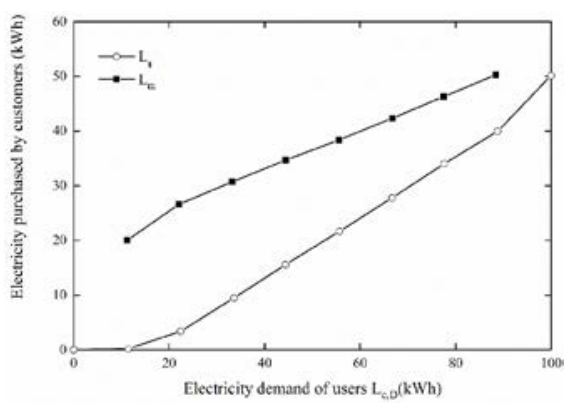

Fig. 2. The trend of power purchases from power companies, photovoltaic power generation and wind power generation networks by users along with changes in user power demand.

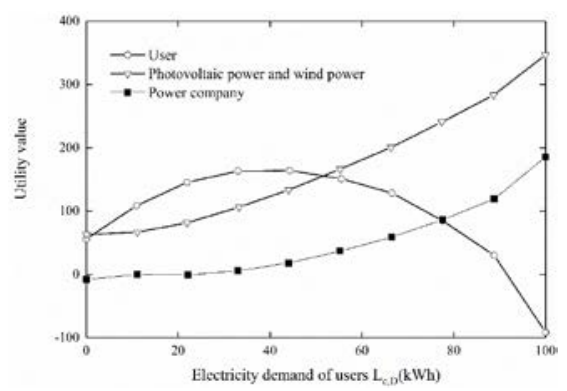

Fig. 3. The utility value of power companies, photovoltaic power generation networks, and users' utility value changes with users' electricity demand. 


\section{Conclusion}

This paper proposes an energy management strategy for photovoltaic power generation and wind power generation networks based on the three-stage Steinberg game. In the first stage, a dominant power company in the system announces its own electricity selling price; in the second stage, multiple photovoltaic and wind power generation networks in the system decide to sell electricity according to the decision of the power company in the first stage Price: In the third stage, users adjust their own electricity demand and announce the amount of electricity demand based on the price of electricity sold by the power company and multiple photovoltaic and wind power generation networks.

The research results of this paper have improved the level of energy services and innovated energy service models. The research results of this paper expand the service business areas and revenues of integrated energy companies, expand the integrated energy service market, and improve the revenue capabilities of integrated energy companies. The research results of this paper have improved the energy service quality and satisfaction of residents. By providing differentiated intelligent energy-using precision services for residents, it can improve the participation and satisfaction of residents' comprehensive energy services and demand response, and solve the problem of high residential customers. The demand for quality services enhances the stickiness of residents. The research results of this article can guarantee economic and social development and improve people's quality of life. Through the analysis and evaluation of household electricity and energy consumption, it provides users with scientific and reasonable energy use suggestions, promotes the scientific and orderly use of electricity by residents, improves energy utilization efficiency, and guarantees sustainable economic and social development; at the same time, clean, A low-carbon, reliable and safe energy supply system will help improve the quality of people's lives.

Research on key technologies, typical demonstrations and business cultivation development paths of the Smart Energy Integrated Service Platform funded by the State Grid Corporation Headquarters Science and Technology Project (5100-201955460A-0-0-00) provided funding for this work.

\section{References}

1. Paras Sitoula, Dwi Rahayu, Pari Delir Haghighi, Sarah Goodwin, and Chris Ling. 2019. Context-Aware Smart Energy Recommender (CASER). In Proceedings of the 17th International Conference on Advances in Mobile Computing \& Multimedia (MoMM2019). Association for Computing Machinery, New York, NY, USA, 13-19. DOI:https://doi.org/10.1145/3365921.3365935.

2. Quanyan Zhu. 2019. Game theory for cyber deception: a tutorial. In Proceedings of the 6th Annual Symposium on Hot Topics in the Science of Security (HotSoS '19). Association for Computing Machinery, New York, NY, USA, Article 8, 1-3. DOI:https://doi.org/10.1145/3314058.3314067.

3. Xiaotong Wu, Genlin Ji, Wanchun Dou, Shui Yu, and Lianyong Qi. 2020. Game Theory for Mobile Location Privacy. In Proceedings of the 2nd ACM International Symposium on Blockchain and Secure Critical Infrastructure (BSCI '20). Association for Computing Machinery, New York, NY, USA, 106-116. DOI:https://doi.org/10.1145/3384943.3409427.

4. Pan Li. 2020. Hybrid Utility Function for Unexpected Recommendations. In Proceedings of the 13th International Conference on Web Search and Data Mining (WSDM '20). Association for Computing Machinery, New York, NY, USA, 923-924. DOI:https://doi.org/10.1145/3336191.3372183. 
5. Xiaozhong Zhang. 2020. Interactive View Recommendation. In Proceedings of the 2020 ACM SIGMOD International Conference on Management of Data (SIGMOD '20). Association for Computing Machinery, New York, NY, USA, 2849-2851. DOI:https://doi.org/10.1145/3318464.3384407.

6. Changhui Yang and Tianyi Sun. 2019. Economic Efficiency Evaluation of Distributed Photovoltaic-Energy Storage Hybrid System Based on the Dynamic Load. In Proceedings of the 2019 2nd International Conference on Information Management and Management Sciences (IMMS 2019). Association for Computing Machinery, New York, NY, USA, 213-218. DOI:https://doi.org/10.1145/3357292.3357331.

7. Eren Yildiz and Kasim Sinan Yildirim. 2020. Defragmenting Energy Storage in Batteryless Sensing Devices. In Proceedings of the 8th International Workshop on Energy Harvesting and Energy-Neutral Sensing Systems (ENSsys '20). Association for Computing Machinery, New York, NY, USA, 36-42. DOI:https://doi.org/10.1145/3417308.3430271.

8. Jiasheng Zhang, Nan Gu, and Chenye Wu. 2020. Energy Storage as Public Asset. In Proceedings of the Eleventh ACM International Conference on Future Energy Systems (e-Energy '20). Association for Computing Machinery, New York, NY, USA, 374-385. DOI:https://doi.org/10.1145/3396851.3397760

9. Vikas Hassija, Vatsal Gupta, Vinay Chamola, and Salil Kanhare. 2020. A blockchainbased framework for energy trading between solar powered base stations and grid. In Proceedings of the Twenty-First International Symposium on Theory, Algorithmic Foundations, and Protocol Design for Mobile Networks and Mobile Computing (Mobihoc '20). Association for Computing Machinery, New York, NY, USA, 315-320. DOI:https://doi.org/10.1145/3397166.3412800 